\title{
MRAS BASED SENSORLESS AND SENSOR VECTOR CONTROL AND SMOOTH TRANSIENT BETWEEN THEM
}

Dynamic control, like vector control is an essential feature of every modern AC drive. In some cases it might be very important to provide continuous operation in case of encoder failure. It further means that the transient between sensor vector control and sensorless vector control has to be smooth. To obtain that a modified vector and sensorless vector control algorithms were designed. To allow smooth transitions, both sensor and sensorless control share common flux model. In this paper a novel robust MRAS-based speed estimator based on error between current estimators output and measured current and modifications of direct sensor vector control are presented. Theoretical analysis of estimators and design of adaptation loop is described. Finally, simulation and experimental results are shown.

\section{Introduction}

As known, induction motors are still the most commonly used motors in the industry, thanks to their good performance and low cost.

Kinds of dynamic control of Induction machines, like Vector Control (Field Oriented Control) or Direct Torque Control have become standard feature of industrial AC drives years ago. Original structures require the using of mechanical speed sensors, but even at that time, the need for mechanical speed sensor elimination was important. During the last two decades many and many different methods and algorithms of speed estimation for vector control have been published; both for induction and synchronous machines [5]. But for high-performance control for speed or position systems still rely on mechanical sensor - quadrature encoder, selsyn, etc. And often it might be important to provide operation even in case of mechanical sensor failure.

In this paper we will focus on direct vector control with sensor feedback and adaptive structures of sensorless vector control Model Reference Adaptive Structure (MRAS). Both of these structures share common flux model for providing continues, smooth transient between sensorless and sensor vector control.

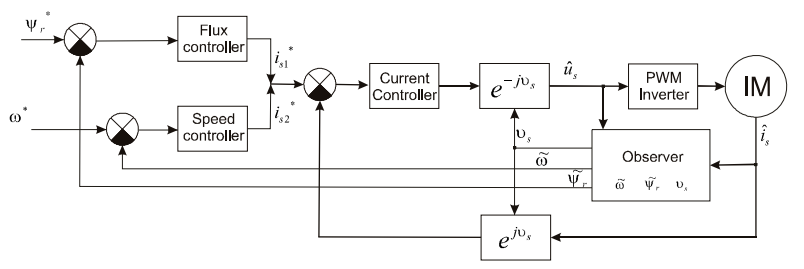

Fig. 1 Basic schema of proposed vector control structure

\section{Sensor vector control}

Proposed sensor vector control is a light modification of "classical" indirect vector control oriented on rotor magnetic flux reference frame to direct vector control. Basic component of proposed control structure is the current model for rotor flux estimation. This model is based on equation originally formulated by Blaschke (often known as Blaschke equation)[1]. This model is mostly used in closed-loop systems where rotor speed is known or at speed close to zero thanks to its reliability. Its main advantage comparing to voltage model of rotor flux is absence of pure integrators.

Current model for flux estimation in stator reference frame $(\alpha, \beta)$ :

$$
s \hat{\psi}_{r}=-\frac{1}{T_{r}} \hat{\psi}_{r}+j \omega \hat{\psi}_{r}+\frac{L_{m}}{T_{r}} \hat{i}_{s}
$$

Current model for flux estimation in reference frame oriented to rotor position $(d, q)$ :

$$
\hat{\psi}_{r}=\frac{L_{m}}{T_{r} s+1} \hat{i}_{s}, T_{r}=\frac{L_{r}}{R_{r}}
$$

Model for current estimation in stator reference frame $(\alpha, \beta)$ :

$$
\hat{i}_{s}=\frac{1}{R_{1}\left(T_{1} s+1\right)}\left(\hat{u}_{s}+\frac{k_{r}}{T_{r}} \hat{\psi}_{r}-j \omega k_{r} \hat{\psi}_{r}\right)
$$

where:

$$
R_{1}=R_{s}+R_{r} k_{r}^{2}, k_{r}=\frac{L_{m}}{L_{r}}, L_{s 1}=\sigma L_{s}, T_{1}=\frac{L_{s 1}}{R_{1}}
$$

Using estimator oriented to rotor position gains several advantages over estimator in stator reference frame. Main advantage is the decoupling of both $(\alpha, \beta)$ components and processing of signals

\footnotetext{
* Jakub Vonkomer, Milan Zalman

Institute of Control and Industrial Informatics, Faculty of Electrical Engineering and Information Technology, Slovak University of Technology in Bratislava, E-mail: jvonkomer@ieee.org
} 


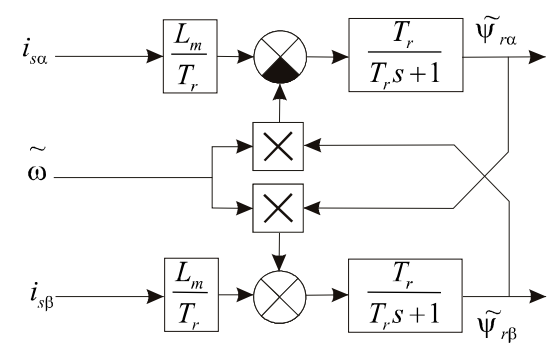

Fig. 2 Flux estimator in stator reference frame $(\alpha, \beta)$

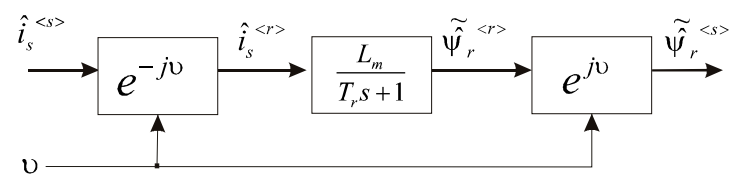

Fig. 3 Flux estimator in reference frame oriented to rotor position $(d, q)$

rotating at the slip frequency only, which is usually not more than a few of Hz. Furthermore park transform is non-dynamical transform.

\section{MRAS Systems}

MRAS (Model Reference Adaptive System) estimators are usually observing mechanical speed by using two different estimators or observers, one of which is speed dependent. Basic schema of MRAS model is shown in Fig. 4.

The difference between estimator outputs is used for speed error reduction, commonly by PID controller, but recently many papers describing adaptation by Fuzzy Logic / Artificial neural networks have been published.

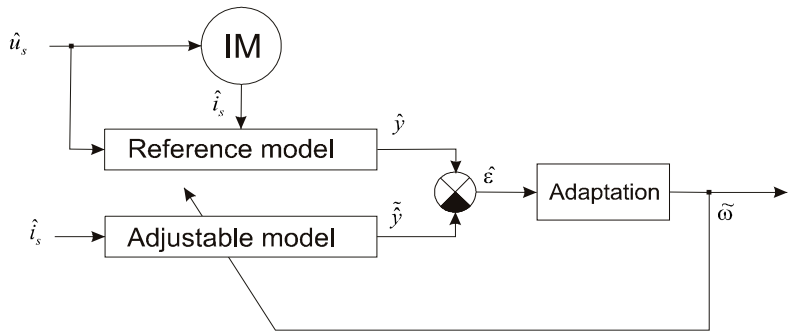

Fig. 4 General schema of MRAS system

There are several groups of MRAS speed estimators. We will focus on the least known structure - structure based on the error of stator current. This estimator was first described by Kowalska [4]. Stator current vector is observed by stator current observers and then compared to measured values. In MRAS terminology, real motor is reference model. As mentioned above, main advantage of this structure is absence of voltage flux model which is unreliable and, therefore unusable at speed close or equal to zero.
Proposed stator current error based MRAS will be later marked as MRASC in this paper.

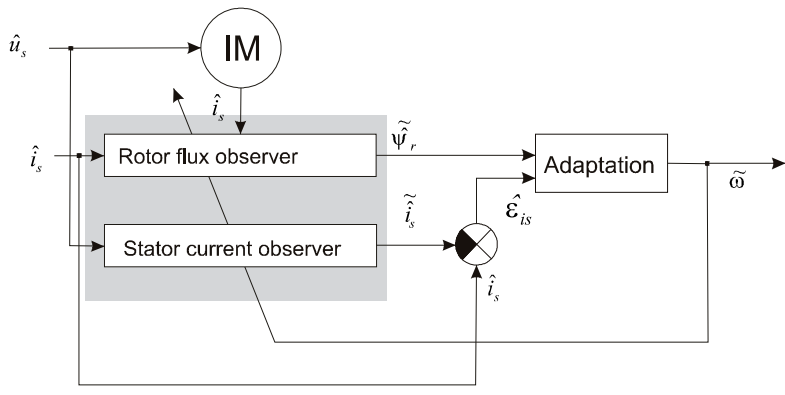

Fig. 5 Simplified schema of proposed MRASC system

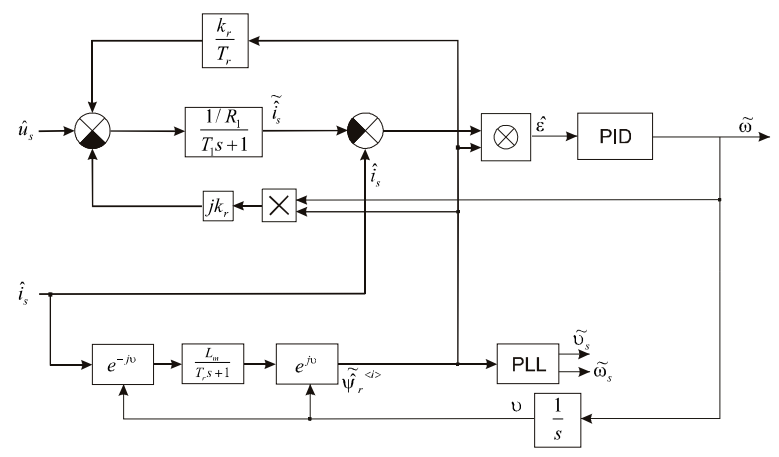

Fig. 6 Detailed schema of proposed MRASC system

Error signal is defined as:

$$
\begin{aligned}
& \varepsilon=\mathfrak{I}\left(\tilde{\hat{\psi}}_{r}^{*} \cdot \hat{e}_{i s}\right)=\left(i_{s \alpha}-\tilde{i}_{s \alpha}\right) \tilde{\psi}_{r \beta}-\left(i_{s \beta}-\tilde{i}_{s \beta}\right) \tilde{\psi}_{r \alpha} \\
& \hat{e}_{i s}=\hat{i}_{s}-\tilde{\hat{i}}_{s}
\end{aligned}
$$

\section{Design of the adaptation loop}

Adaptation loop was designed from the linearized mathematical model.

Under assumption that flux (1) is function of change of speed only (6), flux model equation results to (7).

$\tilde{\hat{\psi}}_{r}=f\left(\tilde{\hat{i}}_{s}, \tilde{\omega}\right) \rightarrow \Delta \tilde{\hat{\psi}}_{r}=f_{1}(\Delta \tilde{\omega})$

$$
\Delta \tilde{\hat{\psi}}_{r}=\frac{j T_{r} \hat{\psi}_{r o}}{\left(T_{r} s+1\right)} \Delta \tilde{\omega}
$$

Model of stator current [2]:

$$
\tilde{\hat{i}}_{s}=\frac{1}{R_{1}\left(T_{1} s+1\right)}\left(\hat{u}_{s}+\frac{k_{r}}{T_{r}} \tilde{\hat{\psi}}_{r}-j \hat{\omega} k_{r} \tilde{\hat{\psi}}_{r}\right)
$$

Now the effect of voltage change is neglected, only flux and speed changes are important: 
$\tilde{\hat{i}}_{s}=f\left(\hat{u}_{s}, \tilde{\hat{\psi}}_{r}, \tilde{\omega}\right) \rightarrow \Delta \tilde{\hat{i}}_{s}=f\left(\Delta \tilde{\hat{\psi}}_{r}, \Delta \tilde{\omega}\right)$

$$
\Delta \tilde{\hat{i}_{s}}=\frac{\frac{k_{r}}{T_{r}} \Delta \tilde{\hat{\psi}}_{r}-j k_{r} \Delta \tilde{\omega} \hat{\psi}_{r o}}{R_{1}\left(1+T_{1} s\right)}
$$

By combining together equations (7) and (10), following results are obtained:

$$
\begin{aligned}
& \Delta \tilde{\hat{i}}_{s}=-\frac{T_{r} s}{R_{1}\left(1+T_{1} s\right)\left(T_{r} s+1\right)} j k_{r} \hat{\psi}_{r o} \Delta \hat{\omega} \\
& \Delta \varepsilon=\Im\left(\hat{\tilde{\psi}}_{r o}^{*} \cdot \Delta \hat{\tilde{e}}_{i s}+\Delta \hat{\tilde{\psi}}_{r}^{*} \cdot \hat{\tilde{e}}_{i s}\right) \\
& \Delta \hat{e}_{i s}=\Delta \hat{i}_{s}-\Delta \tilde{\hat{i}}_{s} \\
& \Delta \varepsilon=-\hat{\psi}_{r o}^{*} \cdot \mathfrak{J}\left(\Delta \hat{e}_{i s}\right)=-\mathfrak{J}\left(\Delta \hat{i}_{s}-\Delta \tilde{\hat{i}}_{s}\right) \hat{\psi}_{r o}^{*} \\
& \Delta \varepsilon=[\Delta \omega-\Delta \tilde{\omega}] \frac{T_{r} s}{\left(1-T_{1} s\right)\left(T_{r} s+1\right)} \frac{k_{r} \hat{\psi}_{r o}^{*}}{R_{1}}
\end{aligned}
$$

\section{Adaptation loop controller}

PID controller was chosen as the most appropriate controller structure. Parameters were designed using pole-placement method. By implementing an integrator into controller structure, simple second order transfer function was obtained.

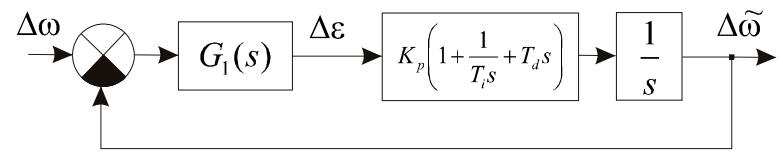

Fig. 7 Control loop for controller design

$$
G_{1}(s)=\frac{\Delta \varepsilon}{\Delta \omega-\Delta \tilde{\omega}}
$$

Final transfer function for calculating the controller parameters:

$$
G_{1}(s)=\frac{K_{0}}{\left(1+T_{1} s\right)\left(T_{r} s+1\right)},
$$

where

$$
K_{0}=\frac{k_{r} \psi_{r o}^{2} T_{r}}{R_{1}}
$$

Closed loop transfer function then results to:

$$
\frac{\Delta \tilde{\omega}}{\Delta \omega}=\frac{\frac{K_{p} K_{0}\left(T_{1} s+1+T_{d} T_{1} s^{2}\right)}{T_{i} T_{1} T_{r}}}{s^{3}+\frac{\left(\left(T_{1}+T_{r}\right)+K_{p} K_{0} T_{d}\right)}{T_{1} T_{r}} s^{2}+\frac{\left(1+K_{p} K_{0}\right)}{T_{1} T_{r}} s+\frac{K_{p} K_{0}}{T_{i} T_{1} T_{r}}}
$$

Reference (characteristic) polynomial:

$$
P_{r e f}=s^{3}+\omega_{0}(2 \zeta+k) s^{2}+\omega_{0}^{2}(2 \zeta k+1) s+k \omega_{0}^{3}
$$

$\omega_{0}$ - system's natural frequency

$\zeta$ - system damping ratio

$k$ - shift pole index

By comparing closed loop denominator polynomial to reference polynomial, parameters for PID were gained.

\section{Transient between sensor and sensorless}

Failure of the encoder can be detected in several ways. The most simple and used in industrial applications is violation of the conditions of complementary signals: $A \neq \bar{A}, B \neq \bar{B}$ etc...

For ensuring the drive operation to continue, the transient to sensorless control must be as smooth as possible. To achieve this when switching from sensor to sensorless control, proper initial values of sensorless control must be set. In our models we assume that the sensorless estimators are in separate block and they are not executed during the sensor control operation. Estimators are executed during the sensorless operation only. In the model of stator current, the discrete filters initial outputs have to be set to actual values of $i_{s \alpha}, i_{s \beta}$ respectively. Otherwise initial value of error signal is unpredictable, thus a strong torque jerk or instability may be caused, especially in high speed region.

The same has to be done in the adaptation PID controller initial output of last integrator has to be set for last known value of electric angular speed from the encoder.

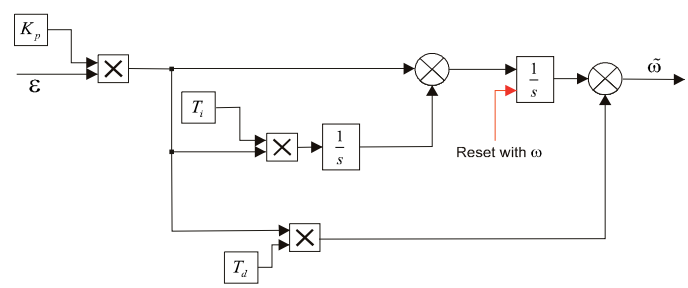

Fig. 8 Structure of MRASC adaptation controller, including RESET signal to achieve smooth transient between sensor and sensorless control

Moreover, it is worth to mention another issue. Usually the knowledge of motor electric parameters for sensor vector control does not need to be as exact as for sensorless vector control. As a result, the transition to sensorless vector control could be unstable. 


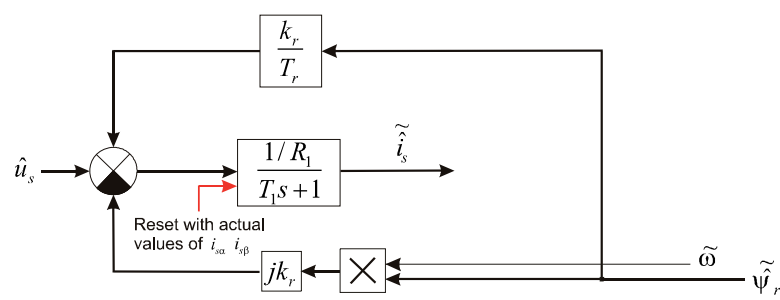

Fig. 9 Schema of model of stator current, including RESET signal to achieve smooth transient between sensor and sensorless control

Therefore, proposed sensorless vector control should be stable and immune against minor parameters variation. To acquire information about this immunity, several experiments have been done in Simulink. By building a close-to-reality simulation model in Simulink with intensive using of SimPowerSystems toolbox blocks, experimental test to parameter variation was done. This test is a complex test of behaviour, it does not only include stability analysis of the adaptation control loop, because the parameter variation affects the other loops, including current and flux controllers as well.

Following tables demonstrate the overall performance and stability of entire systems due to variation of electrical parameters of the motor. These tables differ in dynamics of speed controller (fOSC) and MRAS adaptation controller (f0MRAS).

Regarding the 4 th parameter in the table:

$$
\begin{aligned}
& L_{r}=L_{r \sigma}+L_{m} \\
& L_{s}=L_{r}
\end{aligned}
$$

As might be seen in the previous tables, proposed MRASC does not suffer much from loss of stability or worsen performance caused by small parameter change. Furthermore, this sensitivity is strongly dependent on the dynamics of MRAS adaptation loop speed controller, sample time / PWM frequency, current loop, flux loop and others, so it is hard to present any universally acceptable results. Nevertheless, after implementing simple online estimation algorithms the entire drive performance in the whole speed range could be improved.

\section{Results}

To present excellent behavior of proposed sensor, sensorless control and on-the-run transients between these control methods, both simulation and experimental results are shown below.

\section{Sensorless benchmark test}

Test consists of a simple speed reference signal containing several steps to explore the dynamic properties of sensorless control at various speeds. First is step to $50 \mathrm{~Hz}$, then reverse to $-50 \mathrm{~Hz}$ followed by steps to lower frequencies down to zero.
Experimental analysis of motor's electric

Tab.1 parameters variation
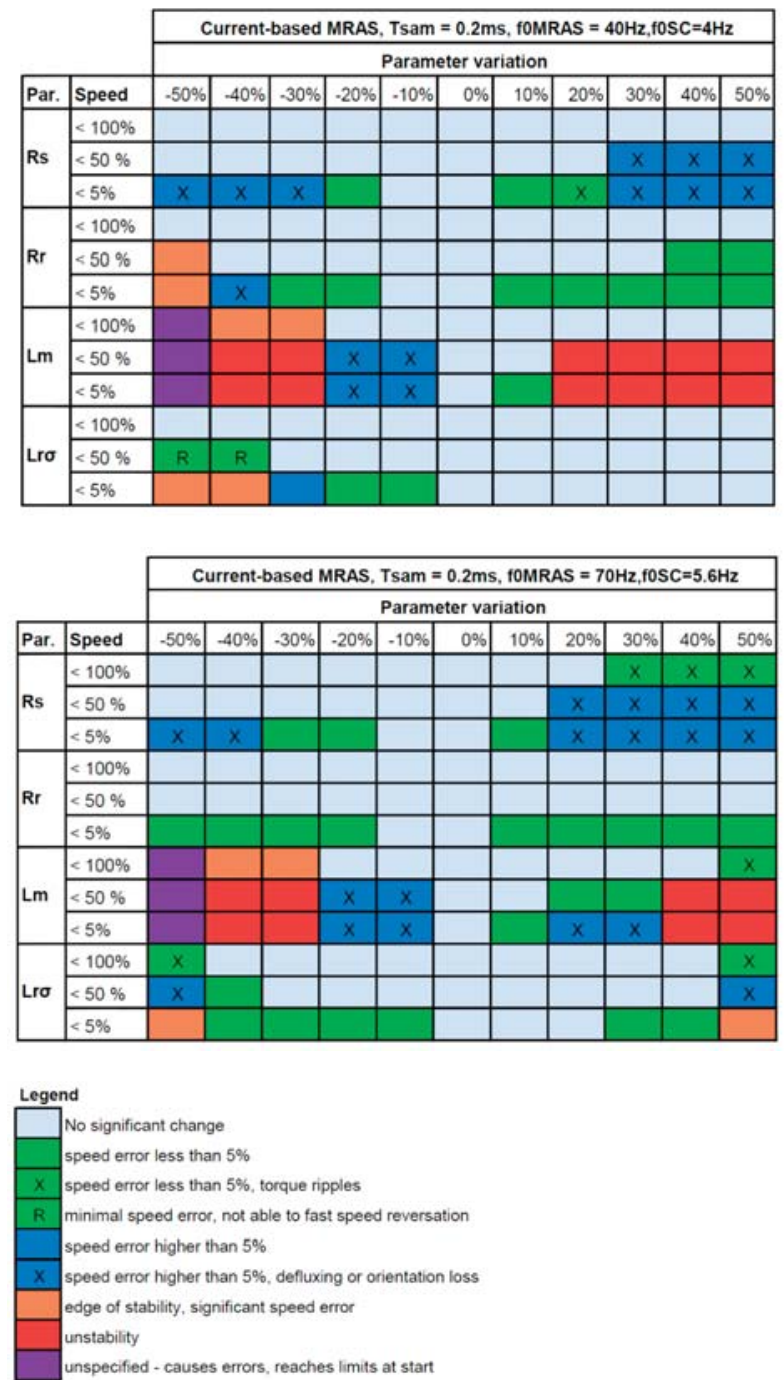

This test shows overall performance of proposed method, including speed reverse possibilities and operation in the low speed region at the end.

Transient from sensor to sensorless control, ramp-up.

This test verifies the quality of transient during the ramp-up. No steady-state for speed reference. Time of transient, $t=0.6 \mathrm{~s}$.

This test also proved very good response, although the dynamics of adaptation is visible on the error between estimated and measured speed. However, the real speed continues smoothly.

Transient from sensor to sensorless control - high speed region

This test had to verify the behaviour of transient from sensor to sensorless control in high speed region under load. This transient is done in $t=2.2 \mathrm{~s}$. 

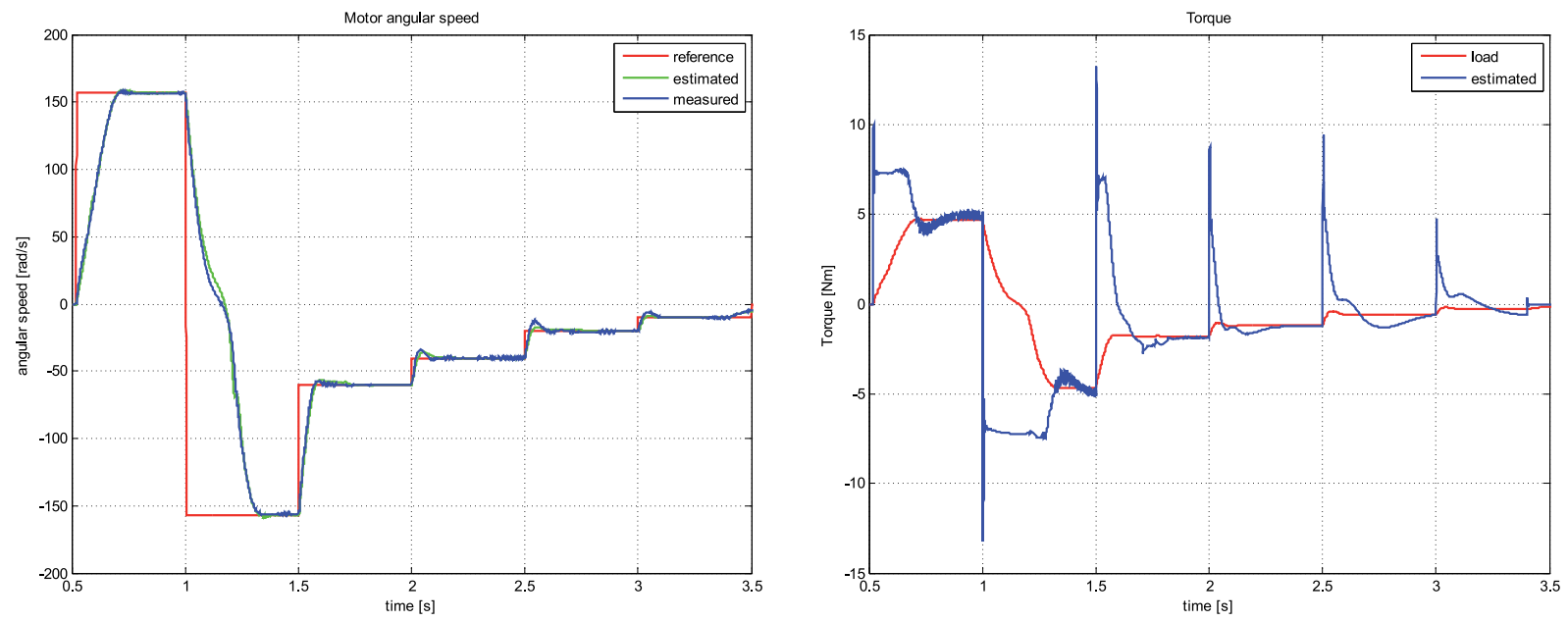

Fig. 10 Sensorless benchmark test: speed and torque
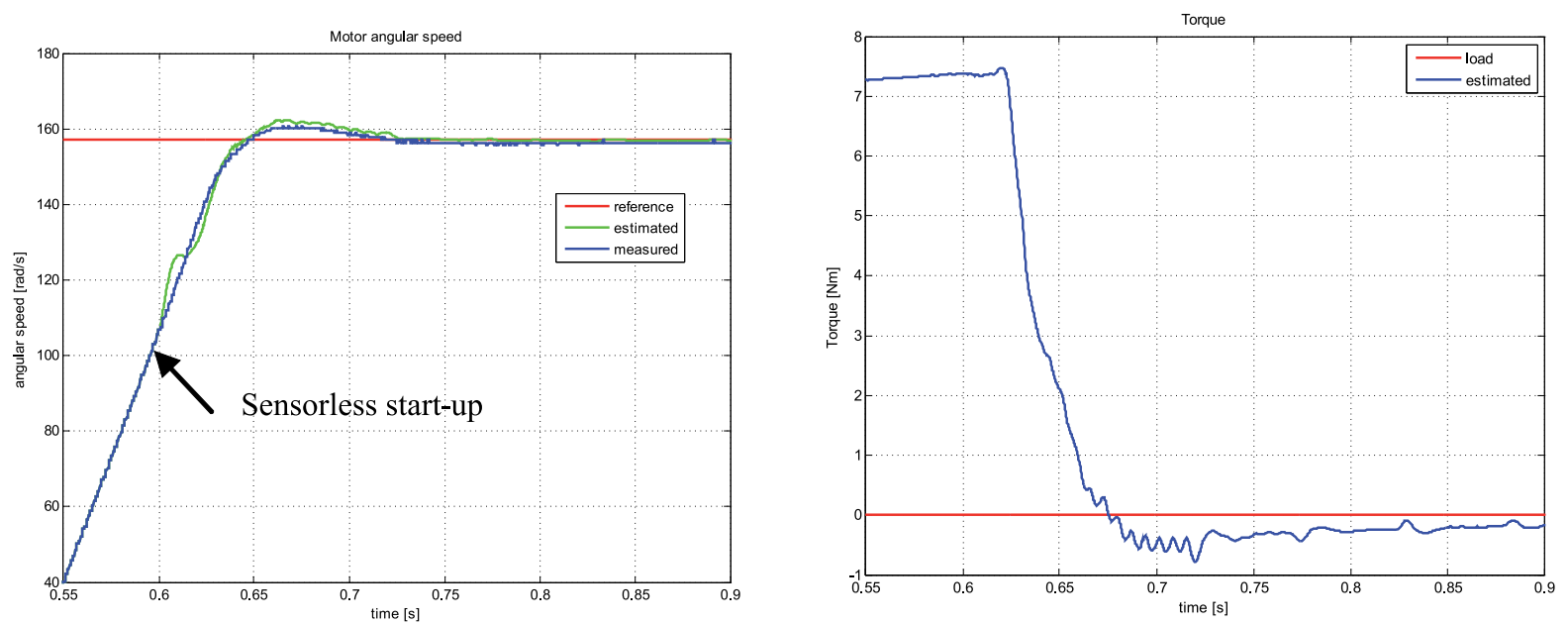

Fig. 11 Ramp-up transient test
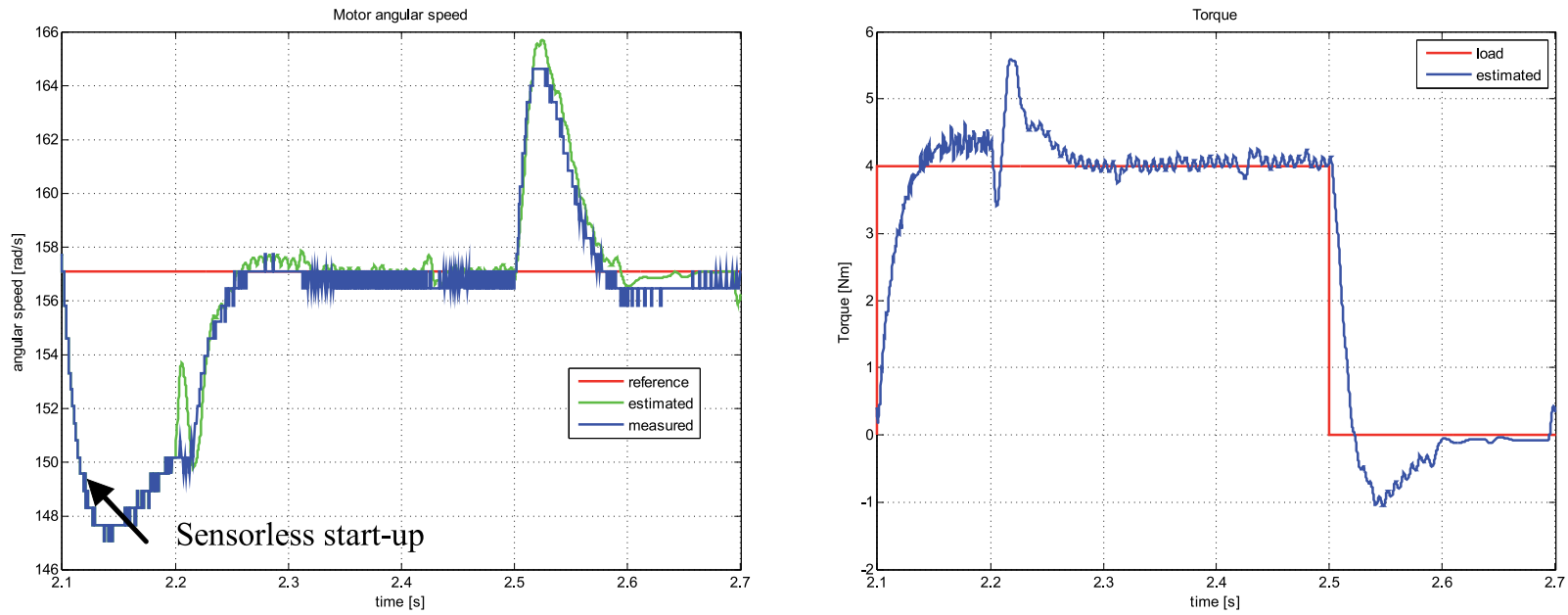

Fig. 12 High speed region transient from sensor to sensorless control, $t=2.2 \mathrm{~s}$ 
Test also showed excellent dynamics and smoothness of the transient between sensor and sensorless control.

\section{Experimental tests:}

Experimental verification was performed in a laboratory setup with PC equipped with dSpace 1104 controller board which controls frequency converter feeding the $1.1 \mathrm{~kW}$ induction machine.

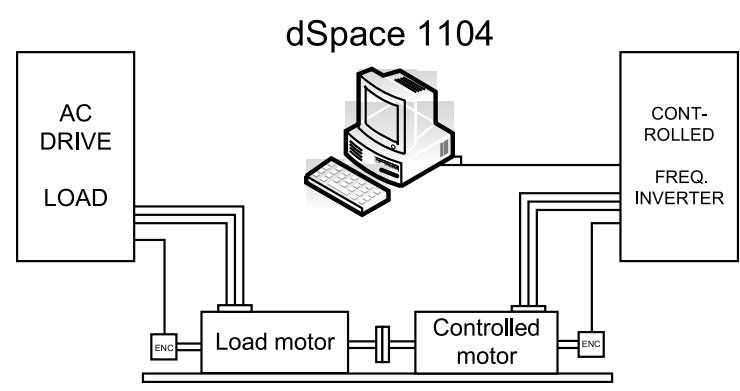

Fig. 13 Diagram of the laboratory test bench

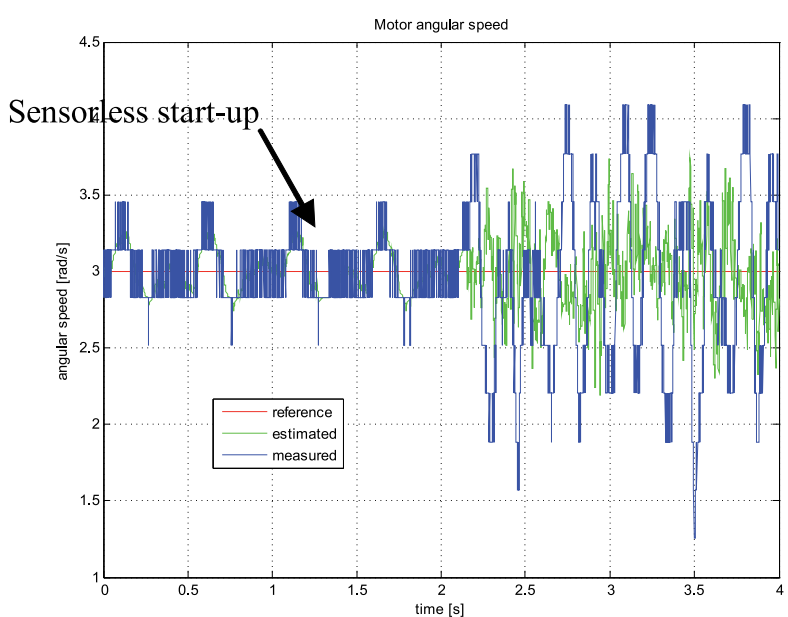

Transient from sensor to sensorless control, low speed region

This test had to verify the behaviour of transient from sensor to sensorless control in low speed region.

Previous test shows non-problematic transient from sensor to sensorless direct vector control.

Transient from sensor to sensorless control, medium speed region

Following test shows the dynamics of a transient in medium speed region. It can be seen that although sensorless control introduces some ripple, the transition itself is smooth.

\section{Conclusion}

The primary objective of our project was to achieve continuous operation of vector control of the drive even in case of encoder's failure. Transient between sensor and sensorless control must be smooth without any significant torque or speed jerk. Moreover, the modified indirect vector control method was described to allow

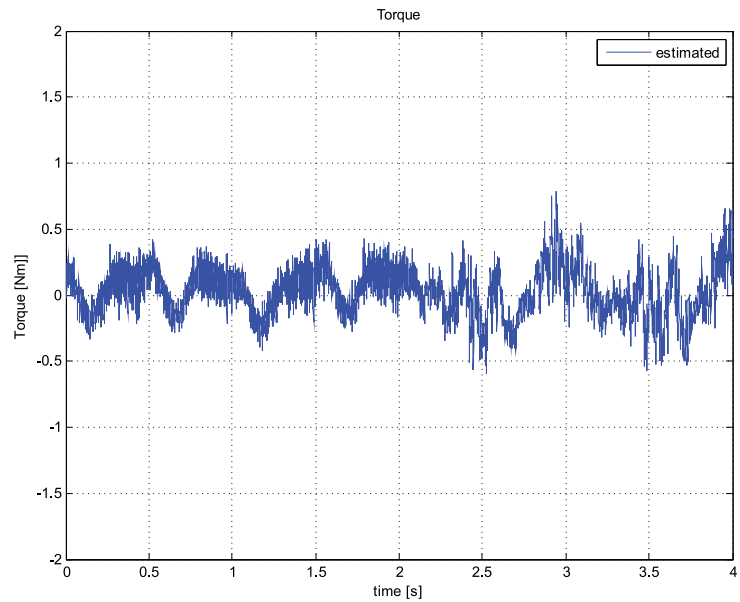

Fig. 14 Low speed experimental results
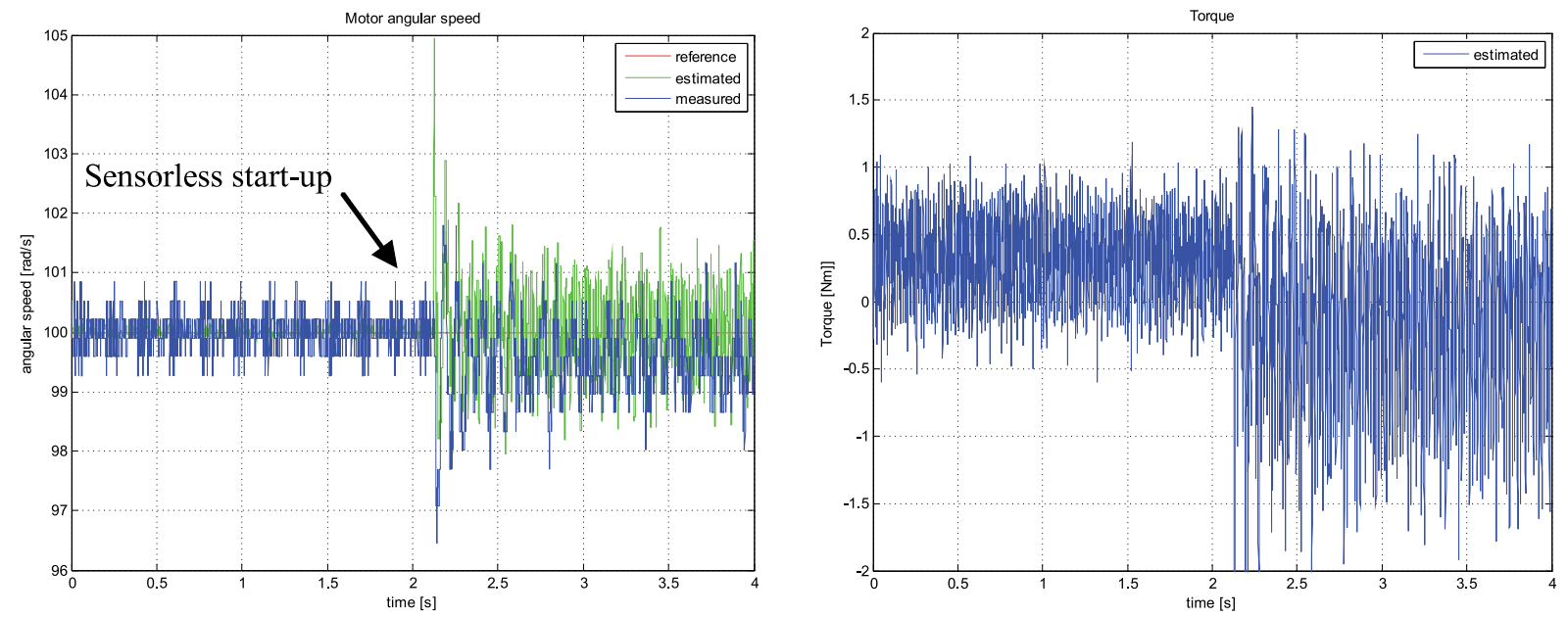

Fig. 15 Medium speed experimental results 
using same flux model with sensorless control. Not very well known approach of MRAS-based sensorless speed control method was demonstrated. Proposed MRASC shows very good dynamic response and appears to be very immune against parameters variation, which is necessary in the industry, where stability and robustness against disturbances is essential. The mathematical model of the estimator including the design of adaptation loop controller was described. Finally, the very good performance of sensorless vector control and the transient between sensor and sensorless vector control were proven by figures.

\section{Acknowledgement}

This work has received support from the Ministry of education, science, research and sport of the Slovak Republic. Project reference number is VEGA $1 / 0690 / 09$.

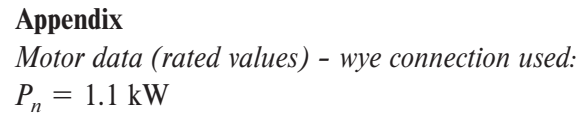

Motor data (rated values) - wye connection used: $P_{n}=1.1 \mathrm{~kW}$

$U_{n}=230 / 400 \mathrm{~V}$ (delta, wye $)$

$I_{n}=5 / 2.9 \mathrm{~A}$

$N_{n}=1380 \mathrm{~min}^{-1}$

Pole pairs $=2$

Electric parameters:

$R_{\mathrm{s}}=7.66 \Omega \quad$ - stator resistance

$R_{r}=5.12 \Omega \quad$ - rotor resistance

$L_{m}=0.386 \mathrm{H} \quad-$ mutual inductance

$L_{r}=0.421 \mathrm{H} \quad$ - rotor inductance

$L_{s}=0.421 \mathrm{H} \quad$ - stator inductance

$J=0.005 \mathrm{~kg} \cdot \mathrm{m}^{2} \quad-$ moment of inertia

Nomenclature

$\hat{x} \quad$ - vectors

$\tilde{x} \quad$ - estimated quantities

\section{References}

[1] BOSE, B. K.; Modern Power Electronics and AC Drives, Prentice Hall, 2002

[2] ZALMAN, M.: The Actuators (in Slovak), STU Bratislava 2003.

[3] ZALMAN, M.: Lectures for Intelligent Servosystems, 2007/2008.

[4] http://servo.urpi.fei.stuba.sk/index.php?option=com_content\&task=view\&id=15\&Itemid=26

[5] DYBKOWSKI, M., ORLOWSKA-KOWALSKA, T.: Application of the Stator Current-based MRAS Speed Estimator in the Sensorless Induction Motor Drive, Power Electronics and Motion Control Conference, 2008. EPE-PEMC 2008. 13th, Vol., No., pp. 2306-2311, 2008, URL: http://ieeexplore.ieee.org/stamp/stamp.jsp?arnumber $=4635607 \&$ isnumber $=4635237$

[6] FILKA, R., BALAZOVIC, P., DOBRUCKY, B.: A Sensorless PM Synchronous Drive for Electric Washers, Communications - Scientific Letters of University of Zilina, No 1., 2007, pp.24-32 URL:http://www.uniza.sk/komunikacie/menu/komunik.asp?id=4\&rok= $2007 \&$ cislo $=1 \& \mathrm{p}=0$. 